\title{
Minimum Path Bases and Relevant Paths
}

\section{Petra M. Gleiss ${ }^{a}$, Josef Leydold $^{b}$, Peter F. Stadler $^{a, c}$}

\author{
${ }^{a}$ Institute for Theoretical Chemistry, \\ University of Vienna, Währingerstrasse 17, A-1090 Vienna, Austria \\ URL: http://www.tbi.univie.ac.at/ ${ }^{\sim p m g}$ \\ ${ }^{b}$ Dept. for Applied Statistics and Data Processing \\ University of Economics and Business Administration \\ Augasse 2-6, A-1090 Wien, Austria \\ Phone: +43 1 31336-4695 Fax: +43 1 31336-738 \\ E-Mail: Josef.Leydold@statistik.wu-wien.ac.at \\ URL: http://statistik.wu-wien.ac.at/ leydold \\ ${ }^{c}$ Bioinformatics Group, Dept. of Computer Science, University of Leipzig \\ Härtelstraße 16-18, D-04107 Leipzig, Germany \\ Phone: +493419716691 Fax: +493419716709 \\ E-Mail: studla@bioinf .uni-leipzig.de \\ URL: http://www.bioinf .uni-leipzig.de/ ${ }^{\sim}$ studla
}

\begin{abstract}
Given an undirected graph $G(V, E)$ and a vertex subset $U \subseteq V$ the $U$ space is the vector space over $G F(2)$ spanned by the paths with end-points in $U$ and the cycles in $G(V, E)$. We extend Vismara's algorithm to the computation of the union of all minimum length bases of the $U$-space. While the size distribution of subgraphs is the same in all minimum length bases, the number of cycles and paths may differ.
\end{abstract}

Keywords: Cycle Space, Minimum Cycle Basis, Relevant Cycles and Paths,

AMS Subject Classification: 05C38 [paths and cycles]. 


\section{Introduction}

In recent years, the structure of a many diverse large "real-world" graphs - including those of social and technological networks, of the metabolic and regulatory networks of a cell, and chemical reaction networks - has been elucidated experimentally, see e.g. [6]. This has lead to a renewed interest in efficient algorithms for computing graph properties that can be used to characterize and classify large network graphs [1].

While most work concentrates on the path structure (which relates directly to transport phenomena in the network), the cycle structure is equally important for an understanding of robustness and resilience since cycles determine the "detours" when edges or vertices are removed [12]. For example, in [4] the distribution is cycle lengths is used to discriminate the off-equilibrium scale-free networks from equilibrium models. In this context short cycles, i.e., short detours, are of particular interest since they convey information on the local ordering in complex networks. Data from real world networks, for instance, reveal the presence of locally grid-like structures through the frequency of quadrangles [7].

From the mathematical point of view, it is natural to consider minimum cycle bases. They have direct applications in diverse areas of science and engineering, such as the static analysis of rigid framework structure [16], chemical structure storage and retrieval systems $[2,9]$, and electric circuit theory [8]. Flux Analysis in chemical reaction networks $[10,20]$ considers stationary fluxes as kernel vector of the stoichiometric matrix, which in the case of a network of isomerization reactions reduces to the incidence matrix of a directed graph. Stationary "flux modes" then reduce to cycles in this graphs [12].

Often, a network contains a special subset $U$ of vertices that e.g. interact with the environment. In such a setting it becomes natural to consider those paths that connect vertices in $U$ with each other in addition to cycles. This becomes particularly apparent for metabolic networks: The nodes in $U$ represent food and waste materials; stationary flux modes then correspond to both cycles and certain paths that begin and end in $U$. The latter form the so-called biochemical pathways on which our understanding of a cell's metabolism is based. In the case of undirected graphs, Hartvigsen's concept of $U$-path bases [13] adds exactly these paths to the cycle space.

A disadvantage of minimum length cycle and path bases, however, is the fact that they are in general not unique. Using the union of all minimum cycle bases solves this problem in many cases. In 1997, Vismara [22] published an efficient algorithm for computing this set of relevant cycles. In this contribution we extend Vismara's algorithm to computing the union of all minimum bases of the $U$-path space and discuss some simple properties of this set.

\section{Preliminaries}

Let $G(V, E)$ be a simple graph with vertex set $V$ and edge set $E$. A cycle in $G$ is a subgraph of $G$ in which each vertex has even degree. A cycle is elementary if it is connected and all its vertices have degree 2. Thus a cycle is an edge-disjoint union of elementary cycles.

A $u v$-path $P, u \neq v$, in $G$ is a connected subgraph that has exactly two vertices of odd degree, $u$ and $v$, called its endnodes, while all other vertices, called the interior 
vertices of $P$ have even degree. A uv-path is elementary if all its interior vertices have degree 2. If a $u v$-path is not elementary, then removing an elementary $u v$-path from it leaves only vertices of even degree, and hence a cycle. Thus a $u v$-path is an edge-disjoint union of an elementary $u v$-path and a collection of elementary cycles.

We reserve the symbol $P_{x y}$ for a shortest path between $x$ and $y$, while $P[x, y]$ denotes the sub-path from $x$ to $y$ of a given elementary path or cycle $P$.

The incidence vector $\vec{H}$ of a subgraph $H$ of $G$ is indexed by the edges of $G$ and has coordinates $H_{e}=1$ if $e$ is an edge of $H$ and $H_{e}=0$ otherwise. We use the same symbol $H$ for a subgraph of $G$, its edge set, and the corresponding incidence vector. It is customary to consider $H$ as a vectors over $G F(2)$. Hence vector addition, $C \oplus D$, corresponds to the symmetric difference of the edge sets of subgraphs $C$ and $D$ of $G$. The incidence vectors of the cycles span the well-known cycle space $\mathfrak{C}(G)$ of $G$, see e.g. [5].

The weight $|H|$ of a subgraph $H$ is simply the number of edges in $H$. The length of a basis $\mathcal{B}$ of a vector space $\mathfrak{V}$ of subgraphs of $G$ is

$$
\ell(\mathcal{B})=\sum_{H \in \mathcal{B}}|H|
$$

We remark that all of the discussion below remains valid when we set $|H|=\sum_{e \in H} \omega(e)$ for arbitrary edge weights $\omega(e)>0$.

\section{U-Path Spaces}

Let $U \subseteq V$ be a nonempty set of vertices, $|U| \geq 2$, and consider the vector space $\mathfrak{U}^{*}$ generated by the incidence vectors of the $u v$-paths, for all $u, v \in U$. This construction is of interest for example in the context of chemical reaction networks, where a subset $U$ of all chemical species $V$ is fed into the system from the outside or is harvested from the system. The $u v$-paths hence correspond to productive pathways [10, 12, 21]. Hartvigsen [13] introduced the $U$-space $\mathfrak{U}(G)$ as the union of $\mathfrak{U}^{*}$ and the cycle space $\mathfrak{C}(G)$. He gives an algorithm for computing a minimum length basis of $\mathfrak{U}(G)$, a minimum $\mathfrak{U}$-basis for short, in polynomial time that extends a previous algorithm by Horton [15] for minimum length bases of the cycle space $\mathfrak{C}(G)$. The following result shows that these two vector spaces are equal on proper graphs.

Lemma 1. If $G$ is biconnected, then $\mathfrak{U}^{*}=\mathfrak{U}(G)$.

Proof. Let $u, v \in U$. Since $\mathfrak{C}(G)$ is spanned by the elementary cycles, it is sufficient to show that any elementary cycle $C$ is the sum of some $u v$-paths. We show that there exist two vertices $x$ and $y$ in $C$, not necessarily distinct, and two paths $D_{1}$ and $D_{2}$ from $u$ to $x$ and from $v$ to $y$, respectively, such that $D_{1}, D_{2}$, and $C$ are edge-disjoint. We also can split the elementary cycle $C$ into two paths $C_{1}$ and $C_{2}$ with $x$ and $y$ as their endnodes, such that $C=C_{1} \oplus C_{2}$. If some of the points $u, v, x$, and $y$ coincide, then some of these paths are empty, i.e. they have no edges. Now $P_{1}=D_{1} \oplus C_{1} \oplus D_{2}$ and $P_{2}=D_{1} \oplus C_{2} \oplus D_{2}$ are two $u v$-paths with $C=P_{1} \oplus P_{2}$, as proposed.

To construct the paths $D_{1}$ and $D_{2}$ we start with an elementary cycle $D$ that contains $u$ and a vertex in $C$. Such a cycle exists by the biconnectedness of $G$. Obviously, $C$ contains two vertices $x$ and $y$ such that the paths $D_{1}=D[u, x]$ and $D_{2}=D[u, y]$ are edge-disjoint and have no edge in common with $C$. Furthermore, since $G$ is connected, 
there must be a path $H$ from $v$ to some vertex $y^{\prime}$ in $C$ that also has no edge in common with $C$. If $D_{1}$ and $H$ are edge-disjoint we can replace $y$ by $y^{\prime}$ and $D_{2}$ by $H$ and we are done. Analogously, we replace $D_{1}$ by $H$ if $D_{2}$ and $H$ are edge-disjoint but $D_{1}$ and $H$ are not. Otherwise $D_{2}$ and $H$ must have a vertex $h$ in common such that the subpath $H[v, h]$ has no edge in common with the sub-paths $D_{2}[h, y]$ and $D_{1}$ (otherwise we change the rôle of $D_{1}$ and $D_{2}$ ). Thus $D_{2}^{\prime}=H[v, h] \oplus D_{2}[h, y]$ is a path from $v$ to $y$ that has no edge in common with $D_{1}$ and $C$. Hence we replace $D_{2}$ by $D_{2}^{\prime}$ and we are done.

Remark. Notice that in the above proof $u$ and $v$ need not be distinct. As a consequence $\mathfrak{C}(G)$ is spanned by all cycles through a given vertex $u \in U$, provide that $G$ is biconnected. It is therefore meaningful to extend the definition of $\mathfrak{U}^{*}$ to the special case $|U|=1$. Then $\mathfrak{U}^{*}$ is the cycle space of the biconnected component that contains $u \in U$. One could therefore define a $u u$-path as a connected cycle through the vertex $u$. However this would complicate the notation in the following.

Remark. Lemma 1 is not necessarily true for general connected graphs. Extending the argument of the proof above it is easy to see, however, that for each biconnected component $H$ of $G$ we have either $\mathfrak{C}(H) \subseteq \mathfrak{U}^{*}$ or $\mathfrak{C}(H) \cap \mathfrak{U}^{*}=\emptyset$, depending on whether a $U$-path passes through $H$.

The $U$-path space $\mathfrak{U}(G)$ of a graph $G(V, E)$ can be transformed into the cycle space of a graph $G^{*}\left(V^{*}, E^{*}\right)$ that is constructed by adding a vertex $z^{*}$ and edges $\left\{z^{*}, u\right\}$ of weight zero for each $u \in U$. $U$-paths then correspond to cycles through $z^{*}$ : every $u v$ path can be transformed into a cycle in $G^{*}$ by adding the path $\left(u, z^{*}, v\right)$; conversely, by deleting $z^{*}$ every cycle containing $z^{*}$ becomes the union of cycles and $u v$-paths in $G$ where $u, v$ are neighbors of $z^{*}$, i.e., a $U$-path. The length of a $u v$-path is obviously equal to the length of the corresponding cycle. As an immediate consequence we get the dimension of the $U$-path space. The dimension of the cycle space of a connected graph is the cyclomatic number $\mu(G)=|E|-|V|+1$. Hence the dimension of the $U$-space is given by $\mu\left(G^{*}\right)=\left|E^{*}\right|-\left|V^{*}\right|+1=(|E|+|U|)-(|V|+1)+1$, see also [14, Prop. 6.5].

Theorem 2. If $G$ is connected then $\operatorname{dim} \mathfrak{U}(G)=\mu(G)+|U|-1=|E|-|V|+|U|$.

Notice that this result also holds for graphs $G$ that are not connected provided that each component of $G$ contains at least one vertex of $U$. In [14] a more general model is considered, where $U_{i}$-paths connect endpoints within one or more disjoint subsets $U_{i} \subset V$. We may deal with this construction in the same way as above by adding a distinct extra vertex for each set $U_{i}$.

\section{Minimum $\mathfrak{U}$-Bases and Relevant $\mathfrak{U}$-Elements}

Definition 3. Let $\mathfrak{V}$ be a vector space of subgraphs of $G$. We say that $A$ is relevant in $\mathfrak{V}$ (for short $\mathfrak{V}$-relevant) if there is a minimum length basis $\mathcal{B}$ of $\mathfrak{V}$ such that $A \in \mathcal{B}$.

In other words, the set of $\mathfrak{V}$-relevant subgraphs is the union of all minimum length bases of $\mathfrak{V}$.

Lemma 4 (Vismara [22]). A $\in \mathfrak{V}$ is relevant if and only if $A$ cannot be written as the $\oplus$-sum of strictly shorter elements of $\mathfrak{V}$. 
Horton's [15] minimum cycle basis algorithm is based on an easy-to-check necessary condition for relevance: A cycle is edge-short if it contains an edge $e=\{x, y\}$ and a vertex $z$ such that $C^{x y, z}=\{x, y\} \cup P_{x z} \cup P_{y z}$ where $P_{x z}$ and $P_{y z}$ are shortest paths. Hartvigsen [13] generalized this notion to paths: A $u v$-path $P$ is edge-short if there is an edge $e=\{x, y\}$ in $P$ such that both $P[u, x]$ is a shortest $u x$-path and $P[y, v]$ is shortest $y v$-path. Horton and Hartvigsen furthermore showed that it is sufficient to consider the cycles $C^{x y, z}$ and paths $P_{u v}^{x, y}=P_{u x} \cup\{x, y\} \cup P_{y v}$ for a fixed choice of the shortest paths $P_{x y}$ between any two vertices of $G$. Thus a minimum cycle basis and a minimum $\mathfrak{U}$-basis can be obtained in polynomial time by means of the greedy algorithm, see $[13,15]$.

A different approach based on the exchange property of matroid is pursued in $[3,18]$. In the case of directed graphs it appears oftentimes more appropriate to consider linear independence over $\mathbb{Q}$ instead of $G F(2)$. In this setting, the minimum cycle basis problem can be solved by analogs of both Horton's and Berger's approaches, see $[19,17]$.

The related problem of computing all relevant cycles or $\mathfrak{U}$-elements can in general not be solved in polynomial time because the number of relevant cycles may grow exponentially with $|V|$ in some graph families, see [22, Fig. 2] for an example. It is possible, however, to define a set of prototypes for the relevant cycles that grows only polynomially with $|V|$. Each relevant cycle $C$ can be then represented in the form

$$
C=C^{p} \oplus S_{1} \oplus S_{2} \oplus \cdots \oplus S_{k}
$$

where $C^{p}$ is a prototype cycle, with $\left|C^{p}\right|=|C|$ and cycles $S_{i}$ that are strictly shorter than $C$. Lemma 4 shows that either both $C$ and $C^{p}$ are relevant or neither cycle is relevant. Furthermore, a minimum cycle basis contains at most one of these two cycles. Vismara [22] describes a construction of prototypes for cycles:

Fix an arbitrary ordering of the vertex set of $G$. Consider an edge-short cycle $C$ such that $r$ is the largest vertex in $C$ w.r.t. this ordering. If there is a vertex $x$ in $C$ such that $C$ consists of two different shortest paths from $x$ to $r$ we say that $C$ is even-balanced. The two vertices adjacent to $x$ in $C$ are denoted by $p$ and $q$. We write $C_{r}^{p x q}$ for this situation. If $C$ contains an edge $\{p, q\}$ such that $\left|P_{p r}\right|,\left|P_{q r}\right|<|C| / 2$ then $C$ is odd-balanced and we write $C_{r}^{p q}$. The cycle family associated with a balanced cycle $C$ consists of all those cycles that share with $C$ the vertex $r$, the edge $\{p, q\}$ or the path $(p, x, q)$, respectively, and that contains shortest paths $P_{p r}$ and $P_{q r}$ such that each vertex in $P_{p r}$ and $P_{q r}$ precedes $r$ in the given ordering. Vismara shows that the members of a cycle family are related by equ.(2) and that the relevant cycle families form a partition of the set of relevant cycles. Any minimum cycle basis contains at most one representative from each cycle family.

This idea can be extended to $U$-paths. Thus the notion of even-balanced and oddbalanced paths is completely analogous and we define the $U$-path family associated with a prototype $P$ as follows:

Definition 5. The $U$-path family $\mathcal{F}(P)$ belonging to the prototype $P$ is the set of all balanced $U$-paths $P^{\prime}$ such that $\left|P^{\prime}\right|=|P|$ and $P^{\prime}$ consists of the vertices $u$ and $v$, the edge $\{x, y\}$ or the path $(x, w, y)$ and two shortest paths $P_{u x}$ and $P_{v y}$.

Hence, two $U$-paths $P$ and $P^{\prime}$ belonging to the same family $\mathcal{F}(P)$ differ only by the shortest paths from $u$ to $x$ and/or from $v$ to $y$ that they include. Consequently, 


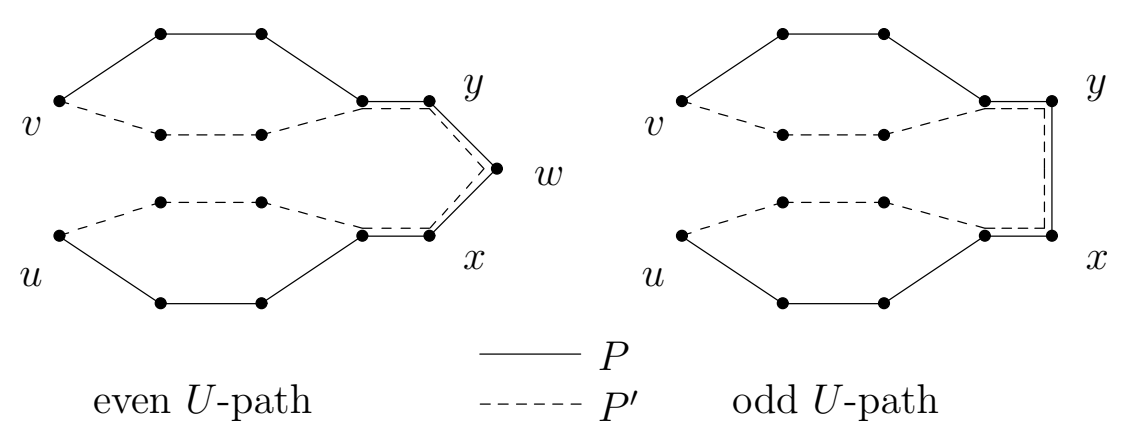

Figure 1

$P=P^{\prime} \oplus S_{1} \oplus S_{2} \oplus \cdots \oplus S_{k}$ where the $S_{j}$ are cycles (or edge-disjoint unions of cycles), see Fig. 1.

The relevant $\mathfrak{U}$-elements can be computed using the two-stage approach proposed by Vismara [22]. This can be done by solving the equivalent Minimum Cycle Basis Problem for the extended graph $G^{*}\left(V^{*}, E^{*}\right)$ but with fixing the additional vertex $z^{*}$ as the largest element in the arbitrary ordering. We do not need to consider all balanced $U$-paths. It is sufficient to consider those that are obtained from balanced cycles through $z^{*}$. The $U$-path prototypes are then obtained by deleting $z^{*}$. Algorithm 1 compiles the necessary steps.

Vismara [22] showed that the prototypes for the relevant cycles can be computed in $\mathcal{O}\left(\mu\left(G^{*}\right)\left|E^{*}\right|^{3}\right)$ steps. Since $|U| \leq|V| \leq|E|$, this is the same as $\mathcal{O}\left((\mu(G)+|U|)|E|^{3}\right)$, i.e., the inclusion of the $U$-paths does not substantially increase the complexity.

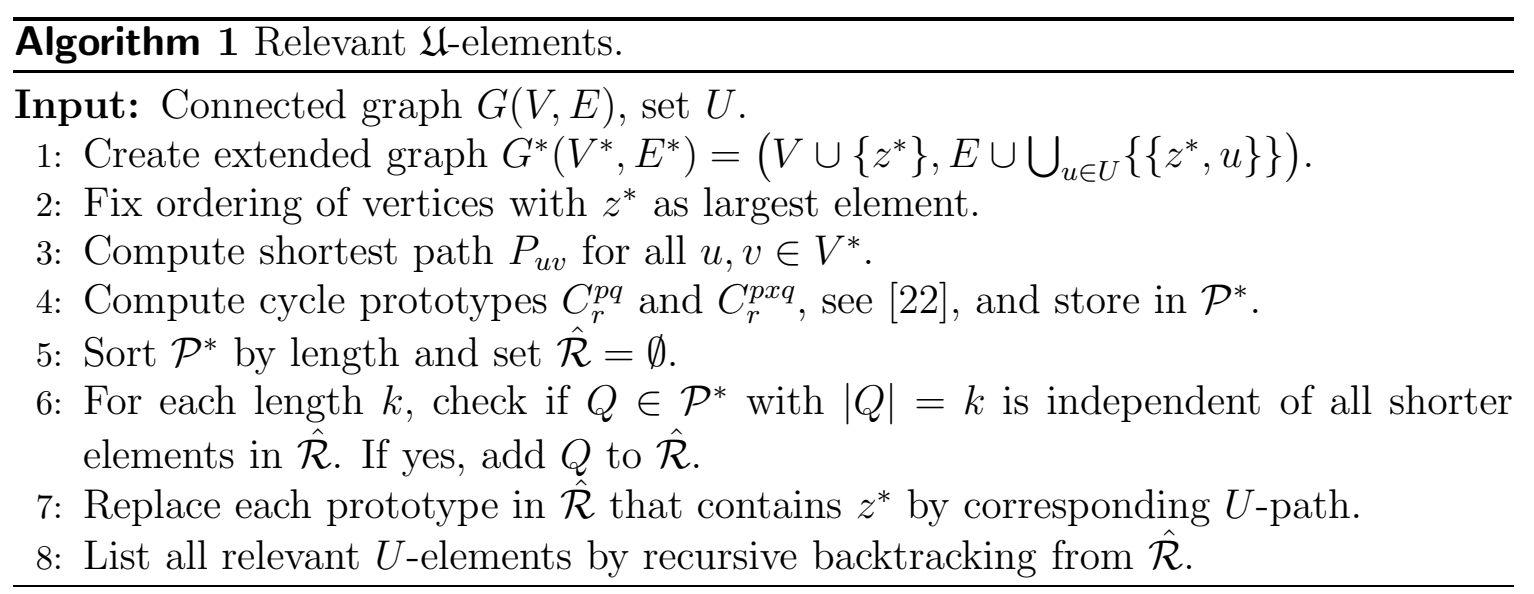
In practice one checks linear independence only against a partial minimum basis.

In the second part the relevant $U$-elements are extracted by means of a recursive backtracking scheme. For each cycle prototype, $C_{r}^{p q}$ (which corresponds to a $U$-path iff $\left.r=z^{*}\right)$ we have to replace the paths $C_{r}^{p q}[p, r]$ and $C_{r}^{p q}[q, r]$ by all possible alternative paths with the same length. These can be generated using the recursive function List_Paths () from [22], where we have to obey additional constraints that $r$ is the vertex with largest index in the given ordering. 


\section{Exchangeability of $\mathfrak{U}$-Elements}

In [11] a partition of the set of relevant cycles is introduced that is coarser than Vismara's cycle families. This construction generalizes directly to the $U$-space:

Definition 6. Two relevant $\mathfrak{U}$-elements $C^{\prime}, C^{\prime \prime} \in \mathcal{R}_{\mathfrak{U}}$ are interchangeable, $C^{\prime} \leftrightarrow C^{\prime \prime}$, if (i) $\left|C^{\prime}\right|=\left|C^{\prime \prime}\right|$ and (ii) there exists a minimum linearly dependent set of relevant $\mathfrak{U}$-elements that contains $C^{\prime}$ and $C^{\prime \prime}$ and has no element that is longer than $C^{\prime}$.

Interchangeability is an equivalence relation. The theory developed in [11] does not depend on the fact that one considers cycles; indeed it works for all finite vector spaces over $G F(2)$ and hence in particular for $U$-spaces. Hence we have the following:

Proposition 7. Let $\mathcal{B}$ be a minimum length $\mathfrak{U}$-basis and let $\mathcal{W}$ be a $\leftrightarrow$-equivalence class of relevant $\mathfrak{U}$-elements. Then $\operatorname{knar}(\mathcal{W})=|\mathcal{W} \cap \mathcal{B}|$ is independent of the choice of the basis $\mathcal{B}$.

The quantity $\operatorname{knar}(\mathcal{W})=|\mathcal{W} \cap \mathcal{B}|$ has been termed the relative rank of the equivalence class $\mathcal{W}$ in [11]. It is tempting to speculate that the $\leftrightarrow$-partition might distinguish between cycles and paths. As the example below shows, however, this is not the case:

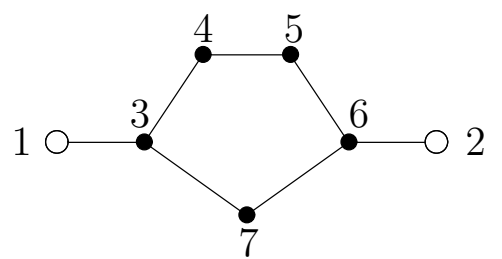

Here $U=\{1,2\}$ and the relevant $\mathfrak{U}$-elements are the paths $P_{1}=(1,3,7,6,2), P_{2}=$ $(1,3,4,5,6,2)$, and the cycle $C=(3,4,5,6,7,3)$. with $\left|P_{1}\right|=4$ and $\left|P_{2}\right|=|C|=5$. Furthermore $C=P_{2} \oplus P_{1}$, i.e., the path $P_{2}$ and the cycle $C$ belong to the same $\leftrightarrow$-equivalence class.

Acknowledgments. We thank two anonymous referees for their valuable comments, which substantially streamlined our arguments. This work was supported by the Austrian Fonds zur Förderung der Wissenschaftlichen Forschung Proj. 14094-MAT and the Bioinformatics Initiative of the Deutsche Forschungs Gemeinschaft, grant no. BIZ-6/1-2.

\section{References}

[1] L. A. N. Amaral, A. Scala, M. Barthélémy, and H. E. Stanley. Classes of small world networks. Proc. Natl. Acad. Sci. USA, 97:11149-11152, 2000.

[2] F. Berger, C. Flamm, P. M. Gleiss, J. Leydold, and P. F. Stadler. Counterexamples in chemical ring perception. J. Chem. Inf. Comput. Sci., 44:323-331, 2004.

[3] F. Berger, P. Gritzmann, and S. de Vries. Minimum cycle bases for network graphs. Algorithmica, 40:51-62, 2004.

[4] G. Bianconi. Number of cycles in off-equilibrium scale-free networks and in the internet at the autonomous system level. Eur. Phys. J. B, 38:223-230, 2004.

[5] B. Bollobás. Modern Graph Theory. Springer, New York, 1998.

[6] S. Bornholdt and H. G. Schuster, editors. Handbook of Graphs and Networks: From the Genome to the Internet. Wiley-VCH, Berlin, 2002. 
[7] G. Caldarelli, R. Pastor-Satorras, and A. Vespignani. Structure of cycles and local ordering in complex networks. Eur. Phys. J. B, 38:183-186, 2004.

[8] L. O. Chua and L. Chen. On optimally sparse cycle and coboundary basis for a linear graph. IEEE Trans. Circuit Theory, 20:54-76, 1973.

[9] G. M. Downs, V. J. Gillet, J. D. Holliday, and M. F. Lynch. Review of ring perception algorithms for chemical graphs. J. Chem. Inf. Comput. Sci., 29:172-187, 1989.

[10] D. A. Fell. Understanding the Control of Metabolism. Portland Press, London, 1997.

[11] P. M. Gleiss, J. Leydold, and P. F. Stadler. Interchangeability of relevant cycles in graphs. Elec. J. Comb., 7:R16 [16pages], 2000.

[12] P. M. Gleiss, P. F. Stadler, A. Wagner, and D. A. Fell. Relevant cycles in chemical reaction network. Adv. Complex Syst., 4:207-226, 2001.

[13] D. Hartvigsen. Minimum path bases. J. Algorithms, 15:125-142, 1993.

[14] D. Hartvigsen. The planar multiterminal cut problem. Discr. Appl. Math., 85:203-222, 1998.

[15] J. D. Horton. A polynomial-time algorithm to find the shortest cycle basis of a graph. SIAM J. Comput., 16:359-366, 1987.

[16] A. Kaveh. Structural Mechanics: Graph and Matrix Methods. Research Studies Press, Exeter, UK, 1992.

[17] T. Kavitha and Mehlhorn. A polynomial time algorithm for minimum cycle basis in directed graphs. In STACS 2005, volume 3404 of Lecture Notes in Computer Science, pages 654-665. Springer, Heidelberg, 2004.

[18] T. Kavitha, K. Mehlhorn, D. Michail, and K. Paluch. A faster algorithm for minimum cycle basis of graphs. In ICALP 2004, volume 3142 of Lecture Notes in Computer Science, pages 846-857. Springer, Heidelberg, 2004.

[19] C. Liebchen and R. Rizzi. A greedy approach to compute a minimum cycle bases of a directed graph. Technical Report 031-2004, TU Berlin, Berlin, Germany, 2004.

[20] C. H. Schilling, D. Letscher, and B. O. Palsson. Theory for systemic definition of metabolic pathways and their use in interpreting metabolic function from pathway-oriented perspective. J. Theor. Biol., 203:229-248, 2000.

[21] O. N. Temkin, A. V. Zeigarnik, and D. Bonchev. Chemical Reaction Networks: A GraphTheoretical Approach. CRC Press, Bocal Raton, FL, 1996.

[22] P. Vismara. Union of all the minimum cycle bases of a graph. Electr. J. Comb., 4(1):73-87, 1997. Paper No. \#R9 (15 pages). 